\title{
Insights into Signaling and the Functional Complexity of Biological Membranes
}

\author{
Leonor Saiz ${ }^{1}$ (1)
}

Received: 21 July 2017/Accepted: 9 August 2017/Published online: 18 August 2017

(C) Springer Science+Business Media, LLC 2017

Biological membranes provide a dynamic platform for signaling mechanisms to process information accurately in the inherently stochastic intra- and extracellular media (Saiz and Vilar 2006). Living systems encounter finite, typically small numbers of signaling molecules that arrive at random times to be sensed by a repertoire of receptors (Mei and Saiz 2014). This is a challenging task in many ways.

There is the obvious sensing of extracellular signals and their intracellular transduction. Receptors need to act cooperatively to perform basic functions, such as detecting thresholds of and changes in ligand concentrations (Nicklas and Saiz 2013; Vilar et al. 2006). This requires a relatively high concentration of different receptors coming together. Biological membranes increase the local concentrations by restricting the motion of the multiple components of the signaling complexes to two dimensions and at the same time they have spatial domains with different properties that localize where these multireceptor assemblies are formed. The domains typically involve different lipid compositions to impart different signaling properties. They affect how receptors interact with each other and how they modulate their allosteric properties.

There are also the physical limitations of the underlying processes. Signal transmission in eukaryotic cells, especially in metazoan cells, cannot rely just on diffusion to propagate across different cellular compartments (Shi and

Leonor Saiz

1saiz@ucdavis.edu

1 Modeling of Biological Networks and Systems Therapeutics Laboratory, Department of Biomedical Engineering, University of California, 451 East Health Sciences Drive, Davis, CA 95616, USA
Massague 2003), from the cell membrane to the nucleus and gene expression (Vilar and Saiz 2013). There are trafficking mechanisms that impart directionality (Vilar and Saiz 2011; Wenk and De Camilli 2004). Signaling complexes respond distinctly depending on the cellular compartment they are located in. Lipid composition of the underlying membranes is a key cue for assembling specific macromolecular signaling complexes in a compartment dependent manner (Le Roy and Wrana 2005; Lemmon 2003).

This special issue features five outstanding pieces on the state of the art of these key aspects of signaling at membranes, covering lipid modifications that change the interactions with proteins (Epand 2016), membrane microdomains that organize signaling networks (Wagner et al. 2016), structural features of lipid-protein interactions of transmembrane receptors (Kalli et al. 2016), protein trafficking and quality control (Printsev et al. 2016), and the effects of the signaling domains in the diffusion of transmembrane receptors in biological membranes (Worch et al. 2016).

In "Features of the Phosphatidylinositol Cycle and its Role in Signal Transduction" (Epand 2016), Rick Epand considers the phosphatidylinositol (PI) cycle, with a special focus on the enzymes responsible for each step and the acyl chain composition of the lipid species. These lipids are essential for signaling in the cell and their different variants are present in different concentrations at both the plasma membrane and the endoplasmic reticulum. The relevance of these systems of lipids is clearly illustrated by changes in acyl chains of PI in cancer cells, which show how key genetic changes can prominently disturb the acyl chain composition of PI. The regulation of many of these lipids affect virtually all signal transduction networks associated 
with the control of cell growth and motion, including apoptosis, proliferation, and migration.

In "Functional Membrane Microdomains Organize Signaling Networks in Bacteria" (Wagner et al. 2016), Daniel Lopez et al. describe our current knowledge on the Bacillus subtilis functional membrane microdomains (FMMs). This emerging field illustrates that bacteria also rely on membrane organization for the proper functioning of fundamental cellular processes in a very similar way as has long been known for eukaryotic cells in processes as diverse as signal transduction, protein sorting, membrane trafficking, or pathogen invasion. The focus is on the scaffold activity of FMMs in stabilizing and promoting the interaction of the signaling components of different membrane-associated signal transduction cascades whose function is linked to the integrity of FMMs.

In "The Integrin Receptor in Biologically Relevant Bilayers: Insights from Molecular Dynamics Simulations" (Kalli et al. 2016), Mark Sansom et al. highlight the role of protein-lipid interactions in integrins, a fundamental class of cell surface receptors that play a fundamental role in cell proliferation and migration. Integrins are potential therapeutic targets in diseases as diverse as cancer, infection, thrombosis, and autoimmune disorders. This study identifies interactions of the receptor with cholesterol and phosphatidylserine lipids that lead to the corralling of the lipids around the receptor. This effect provides a mechanism for regulating the interactions of integrins with other receptor proteins, which facilitates the transduction of extracellular chemical and mechanical signals into intracellular signals.

In "Membrane Protein Quantity Control at the Endoplasmic Reticulum" (Printsev et al. 2016), Kermit Carraway et al. provide a critical discussion of key aspects of the endoplasmic reticulum associated degradation (ERAD) system. The topics covered include the role of the ERAD system as a mechanism for quality control of misfolded proteins, how this system is used for maintaining the proper stoichiometry of multi-protein complexes, and its implications for regulation of plasma membrane-localized signaling receptors. The relevance of this system is illustrated by many explicit examples, including the role of Nrdp1 on the degradation of ErbB3 and its implications for signaling.

In "Diffusion of single-pass transmembrane receptors: from the plasma membrane into giant liposomes" (Worch et al. 2016), Thomas Weidemann et al. describe experiments probing the diffusion behavior of lipids and singlepass transmembrane proteins in the plasma membrane (PM) of living cells, giant plasma membrane vesicles (GPMVs), and giant unilamellar vesicles (GUVs). The key finding is the similarity of diffusion of receptors and lipids in GPMVs and GUVs and their significant difference in the plasma membrane of living cells, indicating that even small protein domains can convey sensitivity to the actin cortex. This type of studies demonstrates how the living cell can modulate the properties of the underlying physical processes.

It has long been established that biological membranes are much more than simple barriers separating different cellular compartments. The topics covered in this special issue on "Signaling at Membranes" illustrate the state of the art in our current understanding on how the structural complexity of biological membranes is coupled to signaling and their fundamental implications.

\section{References}

Epand RM (2016) Features of the phosphatidylinositol cycle and its role in signal transduction. J Membr Biol. doi:10.1007/s00232016-9909-y

Kalli AC, Rog T, Vattulainen I, Campbell ID, Sansom MS (2016) The integrin receptor in biologically relevant bilayers: insights from molecular dynamics simulations. J Membr Biol. doi:10.1007/ s00232-016-9908-z

Le Roy C, Wrana JL (2005) Clathrin- and non-clathrin-mediated endocytic regulation of cell signalling. Nat Rev Mol Cell Biol 6:112-126

Lemmon MA (2003) Phosphoinositide recognition domains. Traffic 4:201-213

Mei Q, Saiz L (2014) Literature-based automated reconstruction, expansion, and refinement of the TGF-beta superfamily ligandreceptor network. J Membr Biol 247:381-386

Nicklas D, Saiz L (2013) Computational modelling of Smad-mediated negative feedback and crosstalk in the TGF-beta superfamily network. J R Soc Interface 10:20130363

Printsev I, Curiel D, Carraway KL 3rd (2016) Membrane protein quantity control at the endoplasmic reticulum. J Membr Biol. doi:10.1007/s00232-016-9931-0

Saiz L, Vilar JMG (2006) Stochastic dynamics of macromolecularassembly networks. Mol Syst Biol 2(2006):0024

Shi Y, Massague J (2003) Mechanisms of TGF-beta signaling from cell membrane to the nucleus. Cell 113:685-700

Vilar JMG, Saiz L (2011) Trafficking coordinate description of intracellular transport control of signaling networks. Biophys J 101:2315-2323

Vilar JMG, Saiz L (2013) Systems biophysics of gene expression. Biophys J 104:2574-2585

Vilar JMG, Jansen R, Sander C (2006) Signal processing in the TGFbeta superfamily ligand-receptor network. PLoS Comput Biol 2:e3

Wagner RM, Kricks L, Lopez D (2016) Functional membrane microdomains organize signaling networks in bacteria. J Membr Biol. doi:10.1007/s00232-016-9923-0

Wenk MR, De Camilli P (2004) Protein-lipid interactions and phosphoinositide metabolism in membrane traffic: insights from vesicle recycling in nerve terminals. Proc Natl Acad Sci USA 101:8262-8269

Worch R, Petrasek Z, Schwille P, Weidemann T (2016) Diffusion of single-pass transmembrane receptors: from the plasma membrane into giant liposomes. J Membr Biol. doi:10.1007/s00232016-9936-8 